\title{
RELACIÓN ENTRE EL NIVEL DE CONOCIMIENTO SOBRE MECANICA CORPORAL Y APLICACIÓN PRACTICA EN ESTUDIANTES DE PRE- GRADO DE LA FACULTAD DE ENFERMERIA DE LA UNIVERSIDAD NACIONAL "SAN LUIS GONZAGA" DE ICA - HOSPITAL SANTA MARIA DEL SOCORRO DE ICA - SETIEMBRE 2009- AGOSTO 2010
}

Relationship between the level of knowledge about body mechanics and practical application in pre-graduate students of the faculty of nursing of the "San Luis Gonzaga" National University of Ica - Hospital Santa Maria of Socorro to Ica - September 2009August 2010

Flora Martha Huisacayna Díaz 1, a,d, Yolanda Aguilar Semino $2, a, b$, Luisa Pacheco VillaGarcia $3, a, c$

\author{
${ }^{1}$ Facultad de enfermería Universidad Nacional "San Luis Gonzaga" de Ica. Perú. \\ ${ }^{2}$ Hospital Santa María del Socorro. Ica, Perú. \\ ${ }^{3}$ Hospital Regional de Ica, Perú \\ a Lic. en Enfermería, ${ }^{b}$ Especialista en Gerencia y Gestión de los Servicios de Salud, ${ }^{\circ}$ Mag en Salud Pública, ${ }^{d}$ Mag en Educación con Mención \\ en Administración y Planificación de la Educación Superior.
}

\section{RESUMEN}

Objetivo: Determinar la relación entre el nivel de conocimiento sobre mecánica corporal y su aplicación práctica en estudiantes de pre- grado de la facultad de enfermería de la Universidad Nacional "San Luis Gonzaga “ de Ica Hospital "Santa María del Socorro" de Ica" setiembre 2009. agosto 2010. Material y Métodos: Enfoque cuantitativo , estudio descriptivo ,de corte transversal y con diseño correlacionar, la muestra fue de 116 estudiantes que realizaron sus prácticas clínicas en el servicio de medicina, y emergencia en el hospital Santa María del Socorro de Ica ,se incluyeron estudiantes que realizaron prácticas clínicas en asignaturas de médico quirúrgico I y urgencias. Utilizando como método la encuesta y observación, como técnica la entrevista y observación directa , para medir conocimiento sobre mecánica corporal se empleó un cuestionario de 20 preguntas, 12 preguntas de selección múltiple y 8 preguntas cerradas, cada ítem tuvo un puntaje de 2 puntos; y para medir la aplicación práctica de mecánica corporal se utilizó una guía de observación de 40 ítems con tres alternativas (siempre, a veces, nunca), que fueron validadas por 4 jueces expertos en el área de Salud Pública y la prueba piloto para obtener su validez y confiabilidad El procesamiento y análisis de los datos fue realizado mediante el uso del método estadístico Ji2 con una probabilidad del 95\% y un margen de error del 5\% la estadística descriptiva fueron tabulados en tabla de Excel y procesados mediante software SPSS Versión 20. Resultados: El 74,14\% de los estudiantes de enfermería de la Universidad Nacional San Luis Gonzaga de Ica, que realizan sus prácticas clínicas en el servicio de medicina y emergencia tienen conocimiento sobre mecánica corporal en un nivel de regular y el 11,21\% con conocimientos buenos. El 72,41\% tuvo una aplicación práctica de mecánica corporal regular y el 10,36\% tuvo una aplicación práctica de buena. Conclusiones: Los niveles de conocimiento sobre mecánica corporal se relacionan con la aplicación práctica de la mecánica corporal de las estudiantes de enfermería de la Universidad Nacional San Luis Gonzaga de Ica

Palabras clave: Conocimiento y práctica Mecánica corporal, estudiantes de enfermería. 


\section{SUMMARY}

Objective: To determine the relationship between the level of knowledge of body mechanics and its practical application in students of pre - degree from the Faculty of nursing of the National University "San Luis Gonzaga" ICA - hospital "Santa Maria of the Socorro of Ica" September 2009 August 2010. Material and methods: It was with quantitative approach, descriptive, cross-sectional, and design study correlate, sample was 116 students conducted their clinical practices in the service of medicine, and emergency hospital Santa Maria del Socorro of Ica and as inclusion criteria: students who performed their clinical practices in the subjects of doctor I surgical and emergency was used as a method the survey and observation, as a technique the entrevista and direct observation, to measure knowledge of body mechanics used a questionnaire of 20 questions, 12 multiple choice questions and 8 -- closed questions, each item. It took a score of 2 points; and a guide to observation of 40 items with three alternatives was used to measure the practical application of body mechanics (always, sometimes, never), which were validated by expert in the area of public health 4 judges and the pilot test to obtain their validity and trust responsibility the processing and analysis of data was carried out using statistical Chi2 method with a probability of $95 \%$ and a margin of error of $5 \%$ descriptive statistics were tabulated in Excel table and processed using the software SPSS Version 20. Results: The $74.14 \%$ of the Universidad Nacional san Luis Gonzaga de Ica nursing students, who perform their clinical practices in medicine and emergency service, have knowledge of body mechanics at a level of regular and the $11.21 \%$ with good knowledge. $72.41 \%$ had a practical application of regular body mechanics and the $10.36 \%$ had an a practical application of good. Conclusions: Levels of knowledge about body mechanics are related to the practical application of the body mechanics of the students of nursing of the National University san Luis Gonzaga de Ica.

Keywords: Knowledge and practice Body mechanics, nursing students.

\section{INTRODUCCION}

Un conocimiento de la mecánica corporal y el uso apropiado del cuerpo son esenciales para muchas actividades cotidianas, ya que en el ámbito de las ciencias de la salud, el personal sanitario tanto profesionales como estudiantes en su mayoría podrían padecer de enfermedades musculo- esqueléticos, lesiones dolorosas, fatiga tensiones, entre otros. La aplicación práctica de los principios de la mecánica corporal, permitirá a dicho personal conservar energías, preservar el tono muscular y la movilidad de las articulaciones y adquirir hábitos de movimientos y elevación que no causen traumatismo a los músculos, ligamentos y articulaciones. Básicamente, la adecuada mecánica corporal es un buen mantenimiento preventivo de cuerpo .Los problemas a consecuencia del uso diario inapropiado de los músculos o aún de una tensión aguda raras veces son inmediatos .La mayoría de las lesiones y problemas son acumulativos y resultan del abuso contante
Aunque la buena mecánica ayuda a una persona a padecer y sentirse mejor, también ayuda a que el trabajo corporal, previniendo de tal modo una calidad asistencial y una disminución del ausentismo laboral (1).

En el trabajo de enfermería se desarrollan múltiples tareas en donde a veces se requiere de un gran esfuerzo físico La movilización y el transporte de pacientes, el trabajo prolongado de pie, los movimientos repetidos y la falta de descanso, constituyen importantes factores de riesgo que producen con el tiempo, lesiones óseas, musculares, articulares y tendinosas. Si a esto se le suma una postura inadecuada y una incorrecta aplicación de los principios de mecánica corporal el riesgo aumenta Enfermería desde el punto de vista epidemiológico es considerada un grupo vulnerable frente a los riesgos laborales, situación que se agrava al no aplicar un buen manejo del cuerpo al momento de realizar cualquier tipo de esfuerzo; entre ellas: lumbalgias, dorsalgias, cervicalgias, trastornos 
neurológicos, etc. La organización Mundial de la salud estima que en América Latina solo se notifican entre $1 \%$ y el $5 \%$ de la enfermedades ocupacionales y que los trabajadores de los servicios hospitalarios están expuestos a una considerable variedad de riesgo (2).

Durante la formación de la enfermera desde que se inicia la carrera se enseña al estudiante las bases fundamentales de la atención del paciente para satisfacer sus necesidades de movimiento, ejercicio, comodidad, reposo higiene etc. Poniendo énfasis en la enseñanza del uso correcto de la mecánica corporal como una manera de promover la salud ocupacional para prevenir problemas de salud Se han realizado varios estudios que hasta ahora, solo han identificado las causas de accidentes de trabajo, factores de riesgo y morbilidad asociadas a la salud ocupacional del personal de enfermería en el medio del hospitalario, pero no se han estudiado cuanto es lo que han aprendido correctamente las enfermeras en su formación sobre la mecánica corporal y como aplican lo aprendido en el ejercicio de su trabajo (3). Las siguientes investigaciones se toman como antecedentes, ya que tienen relación con el trabajo de investigación realizado Un estudio Internacional realizado por: Arteaga D, Pérez N. et.al. 2004 "nivel de conocimiento sobre mecánica corporal y su aplicabilidad en los estudiantes del VI semestre de enfermería U.C.L.A. Decanato de Medicina BARQUISIMETO. Enero- Mayo 2004". Concluye: Sobre el nivel de conocimiento sobre la definición de mecánica corporal el $80 \%$ sabe En cuanto al conocimiento sobre los elementos de la mecánica corporal se identificó sobre alineación corporal el $60 \%$ no sabe; el $65,7 \%$ no conoce la definición de equilibrio, el $40 \%$ no conoce la definición de movimiento corporal coordinado, el $40 \%$ no sabe sobre el principio de mantener el equilibrio y evitar la tensión muscular,34,3\% no conoce el principio de ampliar la base de apoyo y bajar el centro de gravedad , 45,5\% no acertó en el principio de acercar los objetos al centro de gravedad para moverlos , el 74,35 no conoce el principio de mantener el equilibrio con un mínimo esfuerzo .Así mismo el 65,75 no acertó en el principio de contraer los músculos antes de mover un objeto Los resultados y análisis indican que los estudiantes en un gran porcentaje no conoce sobre mecánica corporal; asimismo de acuerdo a la observación y la fotos se evidencian que no aplican los principio de la mecánica corporal(1). Otro estudio realizado por: Pérez S, Sánchez P. 2009 "Riesgos Ergonómicos en las tareas de manipulación de pacientes, en ayudantes de enfermería y auxiliares generales de dos unidades del hospital Clínico de la Universidad de Chile .Concluye: Las tareas "aseo de pacientes, baño en ducha, traslado de gimnasio a cama y medición de pacientes ", tienen un puntaje promedio que corresponde a un nivel de riesgo alto" de padecer un trastorno musculo-esquelético. El nivel de riesgo "alto" de padecer un trastorno musculo- esquelético encontrado en las tareas de las unidades indican una necesidad de intervención pronta y una posterior evaluación (4). Estudio Nacional: por: Carrión E, Huahualuque M. 2003 "Relación entre el nivel de conocimiento sobre mecánica corporal y su aplicación práctica en internas de enfermería, H.R.H.D.2003" Trabajo de investigación con enfoque cuantitativo, descriptivo de corte transversal y con diseño correlacional, se llevó a cabo en el H.R.H.D. Arequipa Concluye: El 8,57 de internas de enfermería poseen un buen nivel de conocimiento sobre mecánica corporal y el 77,14 presenta un nivel de conocimiento Regular; el 14,29 de internas de enfermería tienen una aplicación práctica de la mecánica corporal buena y el 57,14 una aplicación práctica regular (3).

\section{MATERIAL Y METODOS}

El estudio fue cuantitativo, tipo descriptivo, longitudinal, con diseño correlacional. 
La muestra estuvo conformado por 116 estudiantes de enfermería que realizaron sus prácticas clínicas en el Hospital Santa María del Socoro de Ica en los servicios de Medicina y Emergencia.

Se utilizaron como método la encuesta y la observación y como técnica la entrevista y la observación directa.

Previo consentimiento de las estudiantes se les aplico un cuestionario previamente validado por juicios de expertos para medir el nivel de conocimientos sobre mecánica corporal de 20 preguntas, cada ítems tuvo un puntaje de dos puntos, haciendo un puntaje de 40 puntos y para medir la aplicación práctica de mecánica corporal, se utilizó una guía de observación estructurada de 50 ítems, con una dimensión de Siempre 3 puntos a veces 2 puntos Nunca 1 punto.

La observación se hizo después de haber aplicado el cuestionario de conocimientos, la guía de observación se utilizó en dos oportunidades en diferentes días a cada estudiante en forma discreta de manera que la estudiante no se dé cuenta que es observada , para el análisis de la información se utilizó el método estadístico del chi cuadrado con una probabilidad del $95 \%$ y un margen de error del $5 \%$.

La validez se determinó mediante juicios de expertos se hizo uso del coeficiente y la confiabilidad, alfa de Cronbach fue de 0,72 la técnica de análisis e interpretación de datos fue debidamente codificada e ingresada en una base de datos.

Este trabajo se clasificó como investigación sin riesgo para los participantes los cuales fueron identificados con códigos y solo con objetivos de investigación.

\section{RESULTADOS.}

TABLA N ${ }^{\circ}$ 01. LUGAR DONDE REALIZAN SUS PRACTICAS CLINICAS EL GRUPO DE ESTUDIO

\begin{tabular}{ccc}
\hline & \multicolumn{2}{c}{$\begin{array}{c}\text { ESTUDIANTES DE } \\
\text { ENFERMERIA }\end{array}$} \\
\cline { 2 - 3 } SERVICIO & $\mathbf{N}^{\circ}$ & $\%$ \\
\hline MEDICINA & $\mathbf{7 0}$ & 60,35 \\
\hline EMERGENCIA & $\mathbf{4 6}$ & $\mathbf{3 9 , 6 5}$ \\
\hline TOTAL & $\mathbf{1 1 6}$ & $\mathbf{1 0 0 , 0 0}$ \\
\hline FUENTE: INSTRUMENTO APLICADO DE ENERO & A MAYO DEL 2010
\end{tabular}

En la tabla $\mathbf{N}^{\circ} \mathbf{1}$ se observa que el mayor porcentaje de estudiantes del pregrado que están realizando sus prácticas clínicas para el presente estudio están ubicados en el servicio de Medicina

\section{TABLA N 02 NIVEL DE CONOCIMIENTO DE MECANICA} CORPORAL EN EL GRUPO DE ESTUDIO

\begin{tabular}{c|c|c}
\hline \multirow{2}{*}{$\begin{array}{c}\text { NIVEL DE } \\
\text { CONOCIMIENTO }\end{array}$} & \multicolumn{2}{c}{$\begin{array}{c}\text { ESTUDIANTE DE } \\
\text { ENFERMERIA }\end{array}$} \\
\cline { 2 - 3 } & $\mathbf{N}^{\circ}$ & $\%$ \\
\hline MALO & 17 & 14,65 \\
\hline REGULAR & 86 & 74,14 \\
\hline BUENO & 13 & 11,21 \\
\hline TOTAL & 116 & 100,00 \\
\hline Fuente: Ibídem &
\end{tabular}

En la tabla $\mathbf{N}^{\circ} \mathbf{2}$ se observa que del total de la población, el 74,14\% (86 estudiantes) posee conocimientos regulares sobre mecánica corporal, $14,85 \%$ (17 estudiantes) con conocimientos malos y 11,14 \%(13 estudiantes) poseen conocimientos buenos sobre mecánica corporal

\section{TABLA N ${ }^{\circ} 03$ APLICACIÓN PRACTICA DE MECANICA} CORPORAL

\begin{tabular}{ccc}
\hline $\begin{array}{c}\text { APLICACIÓN } \\
\text { PRACTICA } \\
\text { DE MECANICA } \\
\text { CORPORAL }\end{array}$ & \multicolumn{2}{c}{$\begin{array}{c}\text { ESTUDIANTE DE } \\
\text { ENFERMERIA }\end{array}$} \\
\cline { 2 - 3 } & $\mathbf{N}^{\circ}$ & $\%$ \\
\hline MALO & $\mathbf{2 0}$ & $\mathbf{1 7 , 2 3}$ \\
\hline REGULAR & $\mathbf{8 4}$ & $\mathbf{7 2 , 4 1}$ \\
\hline BUENO & 12 & 10,36 \\
\hline TOTAL & 116 & 100,00 \\
\hline Fuente: Ibídem & &
\end{tabular}

Muestra la tabla $\mathbf{N}^{\circ} \mathbf{3}$ del total de las unidades de análisis el 72,41\%(84 estudiantes) tiene una aplicación práctica de la mecánica corporal regular, el 17,23\% (20 estudiantes) con aplicación práctica malo y 10,36\% (12 estudiantes) con aplicación práctica de bueno

TABLA N ${ }^{\circ} 4$ NIVEL DE CONOCIMIENTO, SEGÚN APLICACIÓN PRACTICA EN POSTURA DE PIE

\begin{tabular}{ccccccccc}
\hline & \multicolumn{9}{c}{ CONOCIMIENTO } & \multirow{2}{*}{ TOTAL } \\
\cline { 2 - 8 } $\begin{array}{c}\text { POSTURA } \\
\text { DE PIE }\end{array}$ & \multicolumn{1}{c}{ BUENO } & \multicolumn{2}{c}{ REGULAR } & \multicolumn{2}{c}{ MALO } & & \\
& $\mathbf{N}$ & $\%$ & $\mathbf{N}$ & $\%$ & $\mathbf{N}$ & $\%$ & $\mathbf{N}$ & $\%$ \\
\hline BUENA & $\mathbf{1 1}$ & $\mathbf{9 , 4 8}$ & $\mathbf{2 2}$ & $\mathbf{1 8 . 9 6}$ & $\mathbf{4}$ & $\mathbf{3 , 4 4}$ & $\mathbf{3 7}$ & $\mathbf{3 1 . 8 8}$ \\
\hline REGULAR & $\mathbf{2}$ & $\mathbf{1 , 7 3}$ & $\mathbf{6 0}$ & $\mathbf{5 1 . 7 3}$ & $\mathbf{1 1}$ & $\mathbf{9 , 4 8}$ & $\mathbf{7 3}$ & $\mathbf{6 2 . 9 4}$ \\
\hline
\end{tabular}




\begin{tabular}{ccccccccc|}
\hline MALO & 0 & 00 & 4 & 3.45 & 2 & 1,73 & 6 & 5.18 \\
\hline TOTAL & 13 & 11,21 & 86 & 74.14 & 17 & 14,65 & 116 & 100.00 \\
\hline
\end{tabular}

Fuente: Ibídem

La tabla $\mathbf{N}^{\circ} 4$ presenta que el 9,48\%(11 estudiantes) con conocimiento sobre mecánica corporal bueno, presenta una aplicación práctica de buena en la postura de pie; el $51,73 \%$ (60 estudiantes) con conocimientos regulares de mecánica corporal presenta una aplicación regular en la postura de pie y 9,48\% (11 estudiantes) con conocimientos malos, presenta aplicación regular en la postura de pie

TABLA N`5 NIVEL DE CONOCIMIENTO, SEGÚN APLICACIÓN PRACTICA EN POSTURA AL SENTARSE

\begin{tabular}{|c|c|c|c|c|c|c|c|c|}
\hline \multirow{3}{*}{$\begin{array}{c}\text { POSTURA } \\
\text { AL } \\
\text { SENTARSE }\end{array}$} & \multicolumn{6}{|c|}{ CONOCIMIENTO } & \multirow{2}{*}{\multicolumn{2}{|c|}{ TOTAL }} \\
\hline & \multicolumn{2}{|c|}{ BUENO } & \multicolumn{2}{|c|}{ REGULAR } & \multicolumn{2}{|c|}{ MALO } & & \\
\hline & $\mathbf{N}$ & $\%$ & $\mathbf{N}$ & $\%$ & $\mathbf{N}$ & $\%$ & $\mathbf{N}$ & $\%$ \\
\hline BUENA & 4 & 3,45 & 8 & 6,89 & 2 & 1,73 & 14 & 12,07 \\
\hline REGULAR & 8 & 6,90 & 75 & 64,66 & 11 & 9,47 & 94 & 81,03 \\
\hline MALO & 1 & 0,86 & 3 & & 4 & 3,45 & 8 & 6,9 \\
\hline TOTAL & 13 & 11,21 & 86 & 74,14 & 17 & 14.65 & 116 & 100,00 \\
\hline
\end{tabular}

Fuente: Ibídem

Se observa en la tabla $\mathbf{N}^{\circ} \mathbf{5}$ el ${ }^{\prime} 6,90 \%, 8$ de los estudiantes con conocimientos buenos sobre mecánica corporal, presenta una aplicación práctica de regular en la postura al sentarse; el $64,66 \%$ (75 estudiantes) con conocimientos regulares en mecánica corporal, presentan una aplicación práctica de regular en la postura de sentarse y el 9,47\%(11 estudiantes) con conocimientos malos, presentan una aplicación práctica de regular al sentarse

\begin{tabular}{|c|c|c|c|c|c|c|c|c|}
\hline \multirow{3}{*}{$\begin{array}{c}\text { POSTURA } \\
\text { AL } \\
\text { EMPUJAR } \\
\text { OBJETOS } \\
\text { PESADOS }\end{array}$} & \multicolumn{6}{|c|}{ CONOCIMIENTO } & \multirow{2}{*}{\multicolumn{2}{|c|}{ TOTAL }} \\
\hline & \multicolumn{2}{|c|}{ BUENO } & \multicolumn{2}{|c|}{$\begin{array}{c}\text { REGULA } \\
\mathbf{R} \\
\end{array}$} & \multicolumn{2}{|c|}{ MALO } & & \\
\hline & $\mathbf{N}$ & $\%$ & $\mathbf{N}$ & $\%$ & $\mathbf{N}$ & $\%$ & $\mathbf{N}$ & $\%$ \\
\hline BUENA & 12 & 10,35 & 49 & 42,24 & 10 & 8,62 & 71 & \\
\hline REGULAR & 1 & 0,86 & 33 & 28,45 & 5 & 4,31 & 39 & \\
\hline MALO & 0 & 0,0 & 4 & 3,45 & 2 & 1,72 & 6 & \\
\hline TOTAL & 13 & 11,21 & 86 & 74,14 & 17 & 14,65 & 116 & 100,00 \\
\hline
\end{tabular}

Fuente: Ibídem

La tabla $\mathbf{N}^{\circ} \mathbf{6}$ que el 10,35\%(12) de estudiantes con conocimientos buenos sobre mecánica corporal, presenta una aplicación práctica buena en la postura al empujar objetos pesados; el 42,24\%(49 estudiantes) con conocimientos regulares en mecánica corporal, presentan una aplicación práctica buena en la postura al empujar objetos pesados y el $8,62 \%$ (10 estudiantes) con conocimientos malos, presentan una aplicación práctica de buena al empujar objetos pesados

\section{TABLA N7 NIVEL DE CONOCIMIENTO, SEGÚN APLICACIÓN PRACTICA EN POSTURA AL TRASLADAR PACIENTE DE LA CAMA A CAMILLA}

\begin{tabular}{|c|c|c|c|c|c|c|c|c|}
\hline \multirow{3}{*}{$\begin{array}{c}\text { POSTURA AL } \\
\text { TRASLADAR } \\
\text { PACIENTE DE LA } \\
\text { CAMA A CAMILLA }\end{array}$} & \multicolumn{6}{|c|}{ CONOCIMIENTO } & \multirow{2}{*}{\multicolumn{2}{|c|}{ TOTAL }} \\
\hline & \multicolumn{2}{|c|}{ BUENO } & \multicolumn{2}{|c|}{ REGULAR } & \multicolumn{2}{|c|}{ MALO } & & \\
\hline & $\mathbf{N}$ & $\%$ & $\mathbf{N}$ & $\%$ & $\mathbf{N}$ & $\%$ & $\mathbf{N}$ & $\%$ \\
\hline BUENA & 9 & 7,76 & 59 & 50,86 & 10 & 11,62 & 69 & 70,24 \\
\hline REGULAR & 3 & 2,59 & 20 & 17,25 & 5 & 2,31 & 37 & 22,15 \\
\hline MALO & 1 & 0,86 & 7 & 6,03 & 2 & 0,72 & 10 & 7,61 \\
\hline TOTAL & 13 & 11,21 & 86 & 74,14 & 17 & 14,65 & 116 & 100,00 \\
\hline
\end{tabular}

Se observa en la tabla $\mathbf{N}^{\circ} \mathbf{7}$ que el $7,76 \%, 9$ de los estudiantes con conocimientos buenos sobre mecánica corporal, presenta una aplicación práctica buena en la postura del traslado del paciente de la cama a la camilla; el 50,86\%(59 estudiantes) con conocimientos regulares en mecánica corporal, presentan una aplicación práctica buena en la postura al trasladar paciente de la cama a camilla y el 11,62\%(10 estudiantes ) con conocimientos malos, presentan una aplicación práctica de buena en la postura al trasladar pacientes de la cama a la camilla

TABLA N 8 NIVEL DE CONOCIMIENTO, SEGÚN APLICACIÓN PRACTICA EN POSTURA AL TRASLADAR PACIENTE DE LA CAMA A LA SILLA DE RUEDAS

\begin{tabular}{|c|c|c|c|c|c|c|c|c|}
\hline \multirow{2}{*}{$\begin{array}{l}\text { TRASLADAR } \\
\text { PACIENTE DE } \\
\text { LA CAMA A LA }\end{array}$} & \multicolumn{6}{|c|}{ CONOCIMIENTO } & \multirow{2}{*}{\multicolumn{2}{|c|}{ TOTAL }} \\
\hline & & JENO & REC & țULAR & & ALO & & \\
\hline $\begin{array}{ll}\text { SILLA } & \text { DE } \\
\text { RUEDAS } & \end{array}$ & $\mathbf{N}$ & $\%$ & $\mathbf{N}$ & $\%$ & $\mathbf{N}$ & $\%$ & $\mathbf{N}$ & $\%$ \\
\hline BUENA & 8 & 6,89 & 50 & 43,10 & 10 & 9,62 & 57 & 59,61 \\
\hline REGULAR & 4 & 3,46 & 26 & 22,42 & 5 & 3,31 & 45 & 29,19 \\
\hline MALO & 1 & 0,86 & 10 & 8,62 & 2 & 1,72 & 14 & 11,2 \\
\hline TOTAL & 13 & 11,21 & 86 & 74,14 & 17 & 14,65 & 116 & 100,00 \\
\hline
\end{tabular}

La tabla $\mathbf{N}^{\circ} 8$ se aprecia que el 6,89 \%,8 de los estudiantes con conocimientos buenos sobre mecánica corporal, presenta una aplicación práctica buena en el trasladado del paciente de la cama a la silla de ruedas; el $43,10 \%$ (50 estudiantes) con conocimientos regulares en mecánica corporal, presentan una aplicación práctica de mecánica corporal de buena al trasladar paciente de la cama a la silla de ruedas y el 9,62\%(10 estudiantes) con conocimientos malos, presentan una 
aplicación práctica de buena en la postura al trasladar pacientes de la cama a la silla de rueda.

TABLA N ${ }^{\circ} 9$ NIVEL DE CONOCIMIENTO DE LAS ESTUDIANTES DE ENFERMERIA, SEGÚN APLICACIÓN PRACTICA EN MECANICA CORPORAL - H.S.M.S .ICA 2010

\begin{tabular}{|c|c|c|c|c|c|c|c|c|}
\hline \multirow{3}{*}{$\begin{array}{l}\text { APLICACIÓN } \\
\text { PRÁCTICA DE } \\
\text { MECÁNICA } \\
\text { CORPORAL }\end{array}$} & \multicolumn{6}{|c|}{ CONOCIMIENTO } & \multirow{2}{*}{\multicolumn{2}{|c|}{ TOTAL }} \\
\hline & \multicolumn{2}{|c|}{ BUENO } & \multicolumn{2}{|c|}{ REGULAR } & \multicolumn{2}{|c|}{ MALO } & & \\
\hline & $\mathbf{N}$ & $\%$ & $\mathbf{N}$ & $\%$ & $\mathbf{N}$ & $\%$ & $\mathbf{N}$ & $\%$ \\
\hline BUENA & 9 & 7,77 & 1 & 0,87 & 2 & 1,72 & 12 & 10,36 \\
\hline REGULAR & 2 & 1,72 & 78 & 67,24 & 4 & 3,45 & 84 & 72,41 \\
\hline MALO & 2 & 1,72 & 7 & 6,03 & 11 & 9,48 & 20 & 17,23 \\
\hline TOTAL & 13 & 11,21 & 86 & 74,14 & 17 & 14,65 & 116 & 100,00 \\
\hline
\end{tabular}

Tabla $\mathbf{N}^{\circ} \mathbf{9}$ se observa que las estudiantes que tuvieron conocimientos buenos $11,21 \%$ (13 estudiantes ) ,presentaron en un $7,77 \%$ (9 estudiantes ) buena aplicación de la mecánica corporal; las estudiantes que tuvieron conocimientos regulares en un $74,14 \%$ (86 estudiantes), presentaron en un $67,24 \%$ (78 estudiantes) regular aplicación práctica de la mecánica corporal y las estudiantes que tuvieron conocimientos malos $14,65 \%$ (17 estudiantes), presentaron en un $9,48 \%$ (11 estudiantes) mala aplicación de mecánica corporal

\section{DISCUSIÓN}

En el trabajo de enfermería se desarrollan múltiples tareas en donde a veces se requiere de un gran esfuerzo físico, La movilización y el transporte de pacientes, el trabajo, de pie, los movimientos repetitivos y la falta de descanso constituyen importantes factores de riesgo que producen con el paso del tiempo lesiones óseas, musculares y una incorrecta aplicación de los principios de mecánica corporal el riesgo aumenta (1). La actividad de frecuente manipulación de los pacientes y los elevados niveles de estrés postural a causa de la posición de pie prolongado, posturas inconfortables como por ejemplo torsión o flexión del tronco y la marcha excesiva durante la jornada laboral representa los factores de riesgo fisiológicos o de sobre carga física del personal de enfermería más conocidos (Leighton y Reilly,1995) La realización de las prácticas clínicas pre profesionales es esencial por las estudiantes de enfermería donde pondrán su mejor desempeño en la atención al paciente ,es así, que muchas estudiantes no practican las buenas posturas al dar atención al paciente ; así como al trasladar paciente de la cama a la silla de ruedas, de la cama a la camilla, o viceversa , cuando va a realizar cambios de posición a pacientes postrados, al empujar la camilla , o al sentarse o simplemente al caminar De acuerdo con los resultados obtenidos en la presente investigación en relación .al nivel de conocimiento se observa que el $74,14 \%$ (86 de las estudiantes) tienen conocimientos regulares sobre mecánica corporal, similares resultados se encontraron en la tesis por la bachilleres Carrión E, Huahualuque $M$. "Relación entre el nivel de conocimiento sobre Mecánica Corporal y su aplicación Practica en Internas de Enfermaría , H.R.H.D. 2003" donde refieren que del total de la población un $77,14 \%$ (27estudiantes) poseen conocimientos en un nivel regular (3) , otro trabajo por Arteaga D, Pérez N, Sánchez Á, Silva D " Nivel de conocimiento sobre mecánica corporal y su aplicabilidad en los estudiantes del VI semestre de enfermería U.C.L.A. decanato de Medicina BarquisimetoEnero - Mayo 2004" quienes refieren al mostrar sus resultados : el $80 \%$ no sabe la definición de mecánica corporal, sobre alineación corporal el $60 \%$ no sabe ,el $65,7 \%$ no conocen la definición de equilibrio y el $40 \%$ no conoce la definición de movimiento corporal coordinado (1). En relación a aplicación práctica de mecánica corporal en los estudiantes de enfermería los resultados nos muestra que el $72,41 \%$ (84 estudiantes) tienen una aplicación práctica de mecánica corporal regular, similares resultados se encontraron por Carrión E, et.al. ejecutado en el año 2003 quienes concluyen del total de la población en estudio un 57,14\%(20 estudiantes) tienen una aplicación práctica de mecánica corporal regular (3). .Diferentes resultados se encontraron en un trabajo por Arteaga D.et. al.(2004) indican que la mayoría de estudiantes no conocen sobre los 
principios y directrices de la mecánica corporal , ya que observaron que el $60 \%$ y $70 \%$ de ellos aplican solamente 2 principios de los 11 valorado (1).

\section{Correspondencia:}

Mag. Flora Martha Huisacayna Díaz Correo electrónico: martha.f.h.d@gmail.com

\section{REFERENCIAS BIBLIOGRÁFICAS}

\section{Arteaga D, Pérez Sánchez A, Silva D.} "Nivel de Conocimiento sobre Mecánica Corporal y su Aplicabilidad en los Estudiantes del VI semestre de Enfermería U.C.L.A. Decanato de Medicina Barquisimeto .Enero-Mayo 2004" Tesis Pregrado. Barquisimeto, Venezuela. Programa de Enfermería Decanato de Medicina, Universidad Centroccidental Lisandro Alvarado; 2004.

2. Vega M. "Mecánica Corporal"2009 trabajo de Investigación .Mendoza, Argentina. Universidad del Aconcagua; 2009. p. 1,6

3. Carrión E, Huahualuque M. 2004"Relación entre el Nivel de Conocimiento sobre Mecánica Corporal y su Aplicación Práctica en Internas de Enfermería, H.R.H.D 2003" Tesis Pregrado. Arequipa, Perú. Facultad de Enfermería, Universidad Nacional San Agustín de Arequipa; 2004 .p.71

4. Pérez S, Sánchez P. "Riesgos Ergonómicos en las Tareas de Manipulación de Pacientes, en Ayudantes de Enfermería y Auxiliares Generales de dos Unidades del Hospital Clínico de la
Universidad de Chile" Chile. Tesis Pregrado. Escuela de Kinesiología, Facultad de Medicina, Universidad de Chile; 2009. p.24.

Recibido: 01/09/16

Aprobado para Publicación: 15/01/2017 